\title{
Summary of the various treatments for osteonecrosis of the femoral head by mechanism: A review
}

\author{
CHENG WANG, JIANG PENG and SHIBI LU \\ Institute of Orthopedics, Chinese PLA General Hospital, Beijing 100853, P.R. China \\ Received January 16, 2014; Accepted June 4, 2014
}

DOI: 10.3892/etm.2014.1811

\begin{abstract}
Osteonecrosis of the femoral head (ONFH), also known as femoral head avascular necrosis, is a pathological state with a number of possible etiologies including steroid administration, alcohol abuse, traumatic events, vascular injury and idiopathic origins. ONFH causes a reduction in the vascular supply to the subchondral bone of the femoral head, which results in osteocyte death and the collapse of the articular surface. Treatments for ONFH include non-weight-bearing therapy, physical support, the promotion of osteoclast apoptosis, and the reduction of osteoblast and osteocyte apoptosis. The aim of the present review was to summarize the treatments for ONFH by mechanism from a new perspective and to describe the condition with an emphasis on treatment options.
\end{abstract}

\section{Contents}

1. Introduction

2. Non-weight-bearing (NWB) therapy

3. Physical support

4. Increased osteoclast apoptosis and reduced osteoblast and osteocyte apoptosis

5. Conclusions

\section{Introduction}

Osteonecrosis of the femoral head (ONFH) results in the collapse of the femoral head and the rapid destruction of the hip joint. ONFH has a significant impact on adults aged between 35 and 55 years. This pathological state has several possible etiologies that cause a reduction in the vascular supply to the subchondral bone of the femoral head, resulting in microcirculation disturbance and the subsequent collapse of the femoral head (1-3). In general, the type of treatment carried

Correspondence to: Professor Jiang Peng, Institute of Orthopedics, Chinese PLA General Hospital, 28 Fuxing Road, Haidian, Beijing 100853, P.R. China.

E-mail: pengjiang301@126.com

Key words: osteonecrosis, osteoblast, osteoclast , bisphosphonates out varies according to the disease etiology. Treatments include non-weight-bearing (NWB) therapy, physical support and the alteration of cytoactivity. Extracorporeal shock wave therapy (ESWT) is used to reduce osteoblast and osteocyte apoptosis. Other therapies used include hyperbaric oxygen (HBO) therapy and core decompression. Certain reports (4-6) suggest that bisphosphonates increase osteoclast apoptosis and prevent the collapse of the femoral head. Various femoral head-preserving procedures have been carried out in an attempt to prevent the requirement for a complete hip replacement. The present review focuses on treatments for ONFH $(7,8)$.

\section{NWB therapy}

The initial treatment for early stage ONFH is NWB therapy (9), including a complete rest from weight bearing or changing the weight-bearing status. This type of therapy is able to prevent damage to vessels that supply the femoral head, which is advantageous for ONFH with articular collapse (10). However, a previous review identified that limiting the patient to a wheelchair or walking frame to reduce weight bearing does not achieve satisfactory clinical benefits (11). It was observed that in nonoperatively managed patients with ONFH, only $20 \%$ achieved recovery and $80 \%$ required surgery, including total hip replacement or other treatments (11).

Transtrochanteric rotational osteotomy was developed in $1972(12,13)$ to prevent the progressive collapse of the articular surface and osteoarthritic changes in patients with ONFH. The process is technically demanding and preserves the shape of the femoral head by altering its weight-bearing status (14). However, a number of studies (15-18) have indicated variable rates of success for the surgery. A study by Hiranuma et al (16) evaluated hip instability following transtrochanteric rotational osteotomy. Instability was defined as $>1 \mathrm{~mm}$ translation of the femoral head appearing on transverse computed tomography scans obtained at 0 and $45^{\circ}$ flexion of the hip joint; 11 of the 27 hips studied $(\sim 40 \%)$ revealed instability following surgery.

Although NWB therapy is widely used to treat the early stages of ONFH, certain studies (19-21) have concluded that it is not able to cure the disease or prevent femoral head collapse if it is the only treatment. NWB therapy did not prevent ONFH in rats in a study by Okazaki et al (21), which also clarified the role of weight bearing in the development of ONFH. In the study, an ONFH model was established in non-weight-bearing and weight-bearing groups of rats following lipopolysaccharide 
and methylprednisolone stimulation. Three weeks after the final methylprednisolone injection, the two groups did not differ in the progression of ONFH. Thus, the authors concluded that weight bearing may not contribute to the development of non-traumatic ONFH in rats.

Transtrochanteric rotational osteotomy is an ideal treatment for joint preservation. However, it has not gained widespread acceptance as a treatment for ONFH due to the inconsistency of the obtained results.

\section{Physical support}

\section{Bone grafting.}

The ideal surgical procedure for ONFH would be to remove the necrotic bone from the femoral head and replace it with viable and structurally sound bone, thereby restoring vitality to the femoral head and preventing the collapse of the articular surface. Bone grafting is an appealing treatment option as it combines the benefit of decompressing the femoral head with the introduction of osteoconductive and/or osteoinductive material into the devitalized head. Furthermore, such grafting preserves the natural hip geometry and articular cartilage, unlike the osteotomy and arthroplasty methods.

Nonvascularized and vascularized bone grafting. Nonvascularized bone grafting is an attractive alternative treatment used when there is precollapse or minimal postcollapse of ONFH with relatively preserved articular cartilage (22-27). Rosenwasser et al (28) reported that thorough debridement and cancellous bone-grafting in patients with ONFH was an efficacious treatment, with $87 \%$ of the patients studied remaining essentially free of symptoms and with minimal progression of osteoarthritis following the procedure.

Vascularized bone is grafted onto the necrotic femoral head to directly target its devascularized status. Such a procedure, in addition to replacing necrotic bone with healthy bone, establishes a new source of circulating blood, thus introducing osteoinductive cells and promoting the restoration of a healthy subchondral plate (29-31). Tetik et al (23) carried out a clinical study comparing the results of vascularized and nonvascularized fibular grafting and revealed more improved clinical and radiographic results with vascularized compared with nonvascularized grafting.

Free vascularized fibular grafting has been successfully used as a joint-preserving procedure treatment for ONFH, and a number of studies have demonstrated satisfactory mid- and long-term outcomes (32-36). The procedure may be effective for avoiding or preventing the requirement for complete hip arthroplasty in young patients with early to intermediate stages of ONFH. Kawate et al (37) identified that minor osteonecrosis $\left(<300^{\circ}\right.$ of the femoral head) without preoperative collapse (Steinberg classification stages I and II) was the primary indicator for free vascularized fibular grafting treatment. Osteonecrosis induced by steroid use was a relative counter indicator. Major osteonecrosis $\left(>300^{\circ}\right.$ of the femoral head), with a severe preoperative collapse $(>3 \mathrm{~mm})$, was a major counter indicator. Studies concerning grafting as a treatment for ONFH in patients with lymphoma are rare. The surgical benefits and safety remain to be systematically assessed. Yin et al (38) retrospectively reviewed seven patients (14 hips) with lymphoma (two cases of Hodgkin's disease and five cases of non-Hodgkin's lymphoma) who underwent free vascularized fibular grafting for ONFH. All patients exhibited a positive recovery without any severe life-threatening complications. The mean Harris hip score improved from 69 to 88, while the mean pain score on a visual analog scale decreased from 54 to 18 . Radiography revealed either improved or unchanged results in the three hips with initial Steinberg classification stage II osteonecrosis and in nine of the 11 hips with stage III or IV osteonecrosis. None of the hips demonstrated a failure of the treatment or required complete hip arthroplasty. Despite the technically demanding nature of the procedure, the use of free vascularized fibular grafting improves the quality of life of patients with ONFH, with functional improvement and the alleviation of pain. The procedure involves decompressing the femoral head, excising the necrotic bone and adding a cancellous bone graft with osteoinductive and osteoconductive properties, which augments revascularization and neo-osteogenesis of the femoral head $(37,39)$.

Tantalum implants. Preservation of an osteonecrotic femoral head relies on preventing the collapse of the structurally compromised necrotic bone. Tantalum rod implantation has been proposed as a treatment in the early stages of ONFH. It has demonstrated bone ingrowth and rapid fixation in functional and non-functional animal models and in human explant case reports (40-43). The procedure preserves the structural integrity of the femoral head and is most effective in hips at the pre-collapse stage.

The use of tantalum rods for ONFH was first proposed in 1997 by Schnieders et al (44). The authors used a magnetic resonance-based three-dimensional finite element model of ONFH to study the mechanical effects of inserting a porous tantalum rod into a necrotic femoral head. The porous tantalum rod was a reasonable mechanical substitute for a fibular graft, since it effectively reduced the peak ratio of stress to strain. Liu et al (45) demonstrated that a porous tantalum implant effectively supports the subchondral bone of the femoral head and that the strength of the implant is $>9$-fold than that of the loading stress exerted on the implant. Varitimidis et al (43) studied 27 patients who underwent tantalum rod implantation for nontraumatic ONFH. The implant was a porous tantalum rod $(10 \mathrm{~mm})$. One patient (one hip) succumbed 15 months following surgery for unrelated reasons. In total, 13 of the 26 hips remained at the same radiographic stage, while 13 revealed deterioration. The authors concluded that porous tantalum rods were simple to use via a minimally invasive and reproducible method, and may provide functional recovery for patients at pre- and post-collapse stages of hip osteonecrosis.

These results confirm the curative effects of a porous tantalum rod prosthesis on ONFH, particularly at Steinberg classification stages I and II and at stage IIIA with minimal collapse. The procedure resolves pain as well as preventing and curing the collapse of the femoral head due to necrosis.

\section{Increased osteoclast apoptosis and reduced osteoblast and osteocyte apoptosis}

A number of studies have proposed a balanced system of manipulating osteoclasts and osteoblasts in order to prevent collapse of the femoral head in patients with ONFH $(46,47)$. 
Changes in the prevalence of osteoblast apoptosis may have a significant impact on the number of osteoblasts present at bone formation sites and on their function during therapies including ESWT, HBO treatment and core decompression. Furthermore, an increase in osteoclast apoptosis and inhibited action of mature osteoclasts on bone reduces bone diminution and reinforces bone remodeling (48).

\section{Osteoblast promotion}

ESWT. ESWT began with the incidental observation of the osteoblastic response pattern during animal studies in the mid-1980s, which generated interest in the potential use of ESWT for musculoskeletal disorders (49). Shockwaves are high-energy acoustic waves generated by electrohydraulic or electromagnetic principles. ESWT is effective for treating non-union long bone fractures and tendinopathy of the shoulder, elbow, knee and heel (50) It has been trialed as a treatment for early ONFH and a number of studies (51) have reported the positive effects of the therapy for ONFH $(52,53)$.

Wang et al (52) demonstrated that ESWT is a more effective treatment than core decompression and nonvascularized fibular grafting for patients with early ONFH. In ESWT treatment, the affected hip is positioned in adduction and with internal rotation by securing the limb to a table. For lesions at stages II or III, the junctional zone between the avascular and vascular bone of the femoral head is delineated under C-arm control. Four focal points are selected, $\sim 1.0 \mathrm{~cm}$ apart, and marked with a marker. Each of the four points is treated with 1,500 shock wave impulses at $28 \mathrm{kV}$ (equivalent to $0.6 \mathrm{~mJ} / \mathrm{mm}^{2}$ energy flux density), for a total of 6,000 impulses. Following treatment, patients are instructed to walk on crutches with partial weight bearing on the affected limb for 4-6 weeks. Yin et al (50) reported that ESWT significantly enhanced the angiogenic and osteogenic effects of bone-marrow stem cells via the nitric oxide pathway in patients with ONFH. Thus, ESWT is a novel non-invasive alternative to surgery (which therefore avoids the risks associated with surgery) and is increasingly clinically applied.

HBO therapy. HBO has numerous treatment implications as it has a number of physiological and pharmacological modes of action. Various studies have presented histological evidence that the earliest stage of ONFH is preceded by bone-marrow edema, as observed on magnetic resonance imaging (MRI) scans $(54,55)$. Venous drainage is restored by reducing intraosseous pressure, thereby improving the microcirculation. HBO restores tissue oxygenation, reduces edema and induces angioneogenesis (56). Camporesi et al (57) demonstrated that HBO therapy may be a viable treatment modality for Ficat stage II ONFH. In the study, symptoms were relieved following a multi-year follow-up, without hip arthroplasty being required. In a study by Reis et al (58), 12 patients with Steinberg classification stage 1 ONFH received daily HBO therapy for 100 days. Overall, $81 \%$ of the treated patients demonstrated a normal performance on MRI scans compared with $17 \%$ in the untreated group. The authors thus presented evidence for the benefit of $\mathrm{HBO}$ treatment for idiopathic stage I ONFH. HBO therapy may be useful when carried out in conjunction with core decompression, fenestration and drilling, or other forms of orthopedic intervention. It may also be considered as a primary treatment for ONFH and not merely an adjuvant therapy.
Core decompression. The most popular treatment implemented for ONFH is core decompression, which is the standard surgical procedure carried out to treat early-stage non-traumatic ONFH. Various techniques have been trialed in an attempt to discover a treatment to protect the osteonecrotic femoral head. A number of studies have advocated small-diameter percutaneous drilling (59-61). Mont et al (62) reported a new technique for core decompression involving multiple small drilled holes and a $3-\mathrm{mm}$ Steinman pin. In the study, 32 of the 45 hips (71\%; 35 patients) demonstrated successful clinical results following a mean follow-up of two years (range, 20-39 months). In total, 24 of the 30 stage I hips (80\%; 23 patients) had successful outcomes compared with eight of the 15 stage II hips (57\%; 12 patients), with no surgical complications. This technique may be effective for delaying the need for total hip arthroplasty in young patients with early (precollapse) stages of ONFH. Certain other studies (62-65) have supplemented core decompression with electrical stimulation.

In a study by Steinberg (66), 116 hips with ONFH underwent decompression and grafting; 74 also received direct current stimulation via a coil inserted into the femoral head. Decompression and grafting were safe and reasonably effective for retarding the progression of ONFH. The supplementary electrical stimulation was observed to further improve the results.

Core decompression remains the leading surgical treatment for ONFH (early- and mid-stage), and the efficacy of any new treatments should be compared with core decompression. Marker et al (67) collected data from 1,268 hips following decompression and revealed a clinical success rate of $70 \%$ after 63 months, without the need for additional surgery. Certain studies reported core decompression combined with other treatments produces an improved effect for ONFH (68-70). Implanting a demineralized bone matrix or porous hydroxyapatite composite filler achieves more improved results than using core decompression with other augmentation strategies, including bone marrow and growth factors (71-73).

Adrenocorticotropic hormone (ACTH) and vascular endothelial growth factor $(V E G F)$. The development of $\mathrm{ONFH}$ is accompanied by the apoptosis of osteocytes and osteoclasts (74). Zaidi et al (75) reported that ACTH plays a role in preventing osteonecrosis by promoting osteoblastic support and the expression of VEGF. Early ONFH reveals medium or strong staining of fibroblast growth factor 2 and VEGF, which may promote osteogenesis and bone reconstruction (76).

VEGF is a key factor in bone remodeling. Ingrowth of reparative arterioles has been observed in late-stage osteonecrosis (77). VEGF is a prominent angiogenesis control factor and is used as a therapeutic tool to enhance neovascularization. Angiogenesis is an important part of the bone repair process, and the close connection between blood vessels (endothelial cells) and bone (osteoblasts) was recognized by Trueta and Buhr (78) in 1963. Cao et al (79) implanted deproteinized bone (DPB) with VEGF (absorbed in the form of the recombinant plasmid pcDNA3.1-hVEGF165) into necrotic femoral heads to induce angiogenesis and promote repair. The study revealed that transfection with hVEGF165 enhanced local angiogenesis and the DPB-VEGF compound improved repair. Hang et al (80) evaluated the efficacy of VEGF165 transgenic 


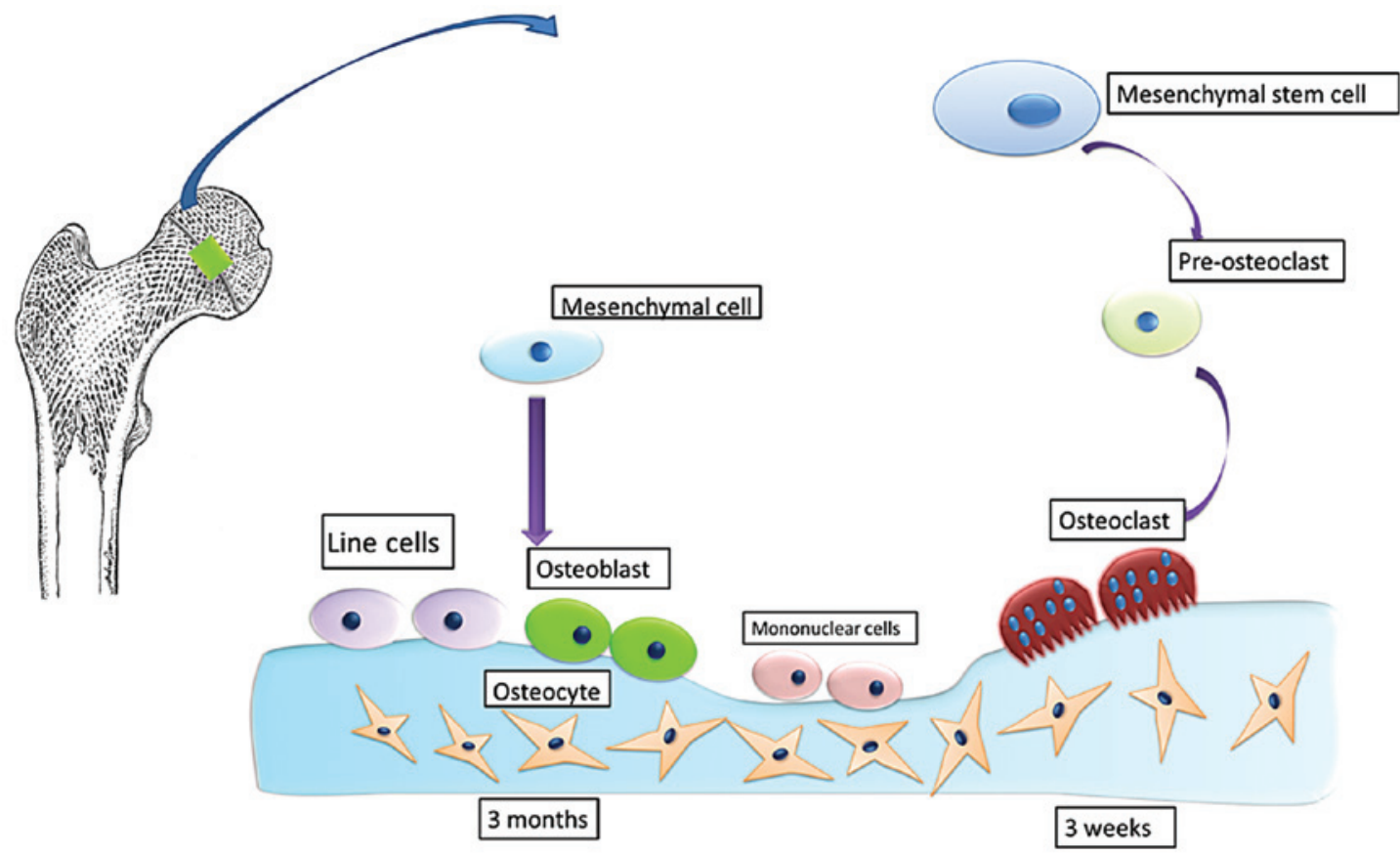

Figure 1. Osteonecrosis of the femoral head. Damage to the bone structure and a reduction in mechanical properties occurs following the beginning of the repair process. It takes $\sim 3$ months to build up new bone with effective mechanical properties, but only $\sim 3$ weeks for osteoclasts to affect the mechanical strength of the trabecular bone. Thus, the repair process inevitably results in the reduction of the mechanical strength of the femoral head. Collapse of the femoral head occurs under the effect of mechanical load.

bone marrow mesenchymal stem cells on the repair of early-stage ONFH in mature mongrel dogs and demonstrated that the treatment enhanced bone reconstruction and blood vessel regeneration.

\section{Osteoclast inhibition}

Pharmacotherapy. Bisphosphonates, such as alendronate, promote osteoclast apoptosis through several mechanisms, including inhibiting protein prenylation and blocking mevalonate metabolism (81). Bisphosphonate reduces edema and the rate of remodeling, which contracts the remodeling spaces and prevents the progression of bone collapse (82). Bone formed during alendronate treatment is histologically normal (83-86) and the treatment appears to offer the greatest protection against fracture. Bisphosphonates are antiresorptive agents that act by inhibiting the action of mature osteoclasts on bone. They transiently stimulate the proliferation and increase the differentiation of pro-osteoblasts, increase the production of the antiresorptive protein osteoprotegerin by osteoblasts, and decrease edema at the site of ONFH, possibly through their anti-inflammatory action.

Agarwala et al (87) treated 69 patients with ONFH with alendronate. The disease duration was 0-36 months. The treatment decreased pain and disability within a few weeks and reduced progressive bone collapse, thus positively altering the progress of ONFH. Alendronate is an antiresorptive agent which reduces edema, the risk of fracture, and the rate of remodeling, thereby contracting the remodeling spaces and preventing the progression of bone collapse.

Although bisphosphonates, including alendronate and zoledronate, have revolutionized treatment for ONFH and osteoporosis, a number of studies (88-94) have demonstrated a serious complication of osteonecrosis in the jaw, which has been the subject of much media attention and resulted in patients at risk of ONFH refusing treatment with bisphosphonates. Outeiriño-Fernández (89) reviewed studies on osteonecrosis of the jaw and concluded that alendronate was the bisphosphonate used in the majority of cases, with a mean duration of treatment prior to jaw osteonecrosis of 48.3 months.

In summary, bisphosphonates have revolutionized the treatment of ONFH; however, they carry an increased risk of bisphosphonate-related osteonecrosis of the jaw. Thus, monitoring should be increased in order to prevent the disadvantages associated with these drugs.

\section{Conclusions}

ONFH is frequently observed in relatively young adults and its management remains controversial. Conservative treatment may be a major focus for orthopedic studies in the future. The principle of the treatment is to provide mechanical support to prevent collapse of the femoral head and improve the speed and quality of repair at the molecular level, increase osteoclast apoptosis, and reduce osteoblast and osteocyte apoptosis. A number of treatment modalities (operative and nonoperative) have positively altered the natural progression of ONFH during the early stage of the disease. Such treatments include ESWT, $\mathrm{HBO}$, pharmacotherapy and paracentesis therapy with VEGF or stem cells. Surgical approaches include core decompression with structural and cellular supplementation. Damage to the bone structure, a reduction in macromechanical performance, and subsequent collapse is considered to occur during the process of restoration. The phenomenon of the destructive bone repair process is inherent, with bone restoration occurring first 
followed by repeated action of the bone leading to fatigue from the load and subsequent fracture. Following the initiation of restoration, neovascularization occurs and new bone formation is required to initially break down the sequestrum. Under continued mechanical loads, the femoral head collapses and there is no further bone resorption or osteogenesis. During the repair process, the balanced bone remodeling carried out by osteoblasts and osteoclasts begins. The process of osteogenesis takes $\sim 3$ months to establish new bone with an effective mechanical performance; however, it takes only $\sim 3$ weeks for osteoclasts to affect the trabecular bone structure. A reduction of the macromechanical strength of the entire femoral head is present in the repair process and also during the collapse of the femoral head under mechanical load. Therefore, delaying or altering the process of femoral head necrosis and collapse through human intervention and recreating a balanced system of osteoblast and osteoclast actions, whilst simultaneously providing sufficient mechanical support, should be studied in the future (Fig. 1).

Preventing collapse of the femoral head is likely to increase in focus in the coming decades since the majority of patients are relatively young at diagnosis. ONFH is undoubtedly a challenging condition to treat; however, ongoing scientific and clinical investigations are progressing towards the development of effective future treatment options.

\section{References}

1. Kim HJ: Autologous adipose tissue-derived stem cells induce persistent bone-like tissue in osteonecrotic femoral heads: not bone-like, but fat-like tissue. Pain Physician 15: E749-E752, 2012.

2. Hasan SS and Romeo AA: Nontraumatic osteonecrosis of the humeral head. J Shoulder Elbow Surg 11: 281-298, 2002.

3. Kaushik AP, Das A and Cui Q: Osteonecrosis of the femoral head: An update in year 2012. World J Orthop 3: 49-57, 2012.

4. Chen $\mathrm{CH}$, Chang JK, Lai KA, Hou SM, Chang CH and Wang GJ: Alendronate in the prevention of collapse of the femoral head in nontraumatic osteonecrosis: a two-year multicenter, prospective, randomized, double-blind, placebo-controlled study. Arthritis Rheum 64: 1572-1578, 2012.

5. Shabtai L, Drexler M and Blummberg N: Biphosphonate in the treatment of avascular necrosis of the femoral head. Harefuah 151: 242-245, 252, 2012 (In Hebrew).

6. Peled E, Bejar J, Barak M, Orion E and Norman D: Core decompression and alendronate treatment of the osteonecrotic rat femoral head: computer-assisted analysis. Int J Exp Pathol 94: 212-216, 2013

7. Amanatullah DF, Strauss EJ and Di Cesare PE: Current management options for osteonecrosis of the femoral head: part II, operative management. Am J Orthop (Belle Mead NJ) 40: E216-E225, 2011.

8. Amanatullah DF, Strauss EJ and Di Cesare PE: Current management options for osteonecrosis of the femoral head: part 1, diagnosis and nonoperative management. Am J Orthop (Belle Mead NJ) 40: E186-E192, 2011.

9. Mihara K and Hirano T: Standing is a causative factor in osteonecrosis of the femoral head in growing rats. J Pediatr Orthop 18: 665-669, 1998

10. Peskin B, Shupak A, Levin D, et al: Effects of non-weight bearing and hyperbaric oxygen therapy in vascular deprivation-induced osteonecrosis of the rat femoral head. Undersea Hyperb Med 28: 187-194, 2001.

11. Mont MA, Carbone JJ and Fairbank AC: Core decompression versus nonoperative management for osteonecrosis of the hip Clin Orthop Relat Res 324: 169-178, 1996.

12. Sugioka Y: Transtrochanteric anterior rotational osteotomy of the femoral head in the treatment of osteonecrosis affecting the hip: a new osteotomy operation. Clin Orthop Relat Res 130: 191-201, 1978.
13. Sugioka Y, Katsuki I and Hotokebuchi T: Transtrochanteric rotational osteotomy of the femoral head for the treatment of osteonecrosis. Follow-up statistics. Clin Orthop Relat Res 169: 115-126, 1982.

14. Koo KH, Song HR, Yang JW, Yang P, Kim JR and Kim YM: Trochanteric rotational osteotomy for osteonecrosis of the femoral head. J Bone Joint Surg Br 83: 83-89, 2001.

15. Eyb R and Kotz R: The transtrochanteric anterior rotational osteotomy of Sugioka. Early and late results in idiopathic aseptic femoral head necrosis. Arch Orthop Trauma Surg 106: 161-167, 1987.

16. Hiranuma Y, Atsumi T, Kajiwara T, Tamaoki S and Asakura Y: Evaluation of instability after transtrochanteric anterior rotational osteotomy for nontraumatic osteonecrosis of the femoral head. J Orthop Sci 14: 535-542, 2009.

17. Yamamoto T, Ikemura S, Iwamoto $Y$ and Sugioka Y: The repair process of osteonecrosis after a transtrochanteric rotational osteotomy. Clin Orthop Relat Res 468: 3186-3191, 2010.

18. Dean MT and Cabanela ME: Transtrochanteric anterior rotational osteotomy for avascular necrosis of the femoral head. Long-term results. J Bone Joint Surg Br 75: 597-601, 1993.

19. Mont MA and Hungerford DS: Non-traumatic avascular necrosis of the femoral head. J Bone Joint Surg Am 77: 459-474, 1995.

20. Lavernia CJ, Sierra RJ and Grieco FR: Osteonecrosis of the femoral head. J Am Acad Orthop Surg 7: 250-261, 1999.

21. Okazaki S, Nagoya S, Tateda K, et al: Weight bearing does not contribute to the development of osteonecrosis of the femoral head. Int J Exp Pathol 93: 458-462, 2012.

22. Ohzono K, Saito M, Takaoka K, et al: Natural history of nontraumatic avascular necrosis of the femoral head. J Bone Joint Surg Br 73: 68-72, 1991.

23. Tetik C, Başar H, Bezer M, Erol B, Ağir I and Esemenli T: Comparison of early results of vascularized and non-vascularized fibular grafting in the treatment of osteonecrosis of the femoral head. Acta Orthop Traumatol Turc 45: 326-334, 2011.

24. Seyler TM, Marker DR, Ulrich SD, Fatscher T and Mont MA: Nonvascularized bone grafting defers joint arthroplasty in hip osteonecrosis. Clin Orthop Relat Res 466: 1125-1132, 2008.

25. Mont MA, Marulanda GA, Seyler TM, Plate JF and Delanois RE: Core decompression and nonvascularized bone grafting for the treatment of early stage osteonecrosis of the femoral head. Instr Course Lect 56: 213-220, 2007.

26. Kim SY, Kim YG, Kim PT, Ihn JC, Cho BC and Koo KH: Vascularized compared with nonvascularized fibular grafts for large osteonecrotic lesions of the femoral head. J Bone Joint Surg Am 87: 2012-2018, 2005.

27. Mont MA, Etienne G and Ragland PS: Outcome of nonvascularized bone grafting for osteonecrosis of the femoral head. Clin Orthop Relat Res 417: 84-92, 2003.

28. Rosenwasser MP, Garino JP, Kiernan HA and Michelsen CB: Long term followup of thorough debridement and cancellous bone grafting of the femoral head for avascular necrosis. Clin Orthop Relat Res 306: 17-27, 1994.

29. Soucacos PN, Beris AE, Malizos K, Koropilias A, Zalavras H and Dailiana Z: Treatment of avascular necrosis of the femoral head with vascularized fibular transplant. Clin Orthop Relat Res 386: 120-130, 2001.

30. Marciniak D, Furey C and Shaffer JW: Osteonecrosis of the femoral head. A study of 101 hips treated with vascularized fibular grafting. J Bone Joint Surg Am 87: 742-747, 2005.

31. Malizos KN, Soucacos PN and Beris AE: Osteonecrosis of the femoral head. Hip salvaging with implantation of a vascularized fibular graft. Clin Orthop Relat Res 314: 67-75, 1995.

32. Sakamoto A, Yoshida T, Uchida Y, Kojima T, Kubota H and Iwamoto Y: Long-term follow-up on the use of vascularized fibular graft for the treatment of congenital pseudarthrosis of the tibia. J Orthop Surg Res 3: 13, 2008.

33. Zhang C, Zeng B, Xu Z, et al: Treatment of femoral head necrosis with free vascularized fibula grafting: a preliminary report. Microsurgery 25: 305-309, 2005.

34. Berend KR, Gunneson EE and Urbaniak JR: Free vascularized fibular grafting for the treatment of postcollapse osteonecrosis of the femoral head. J Bone Joint Surg Am 85-A: 987-993, 2003.

35. Liang K, Xiang Z, Chen S, et al: Folded free vascularized fibular grafts for the treatment of subtrochanteric fractures complicated with segmental bone defects. J Trauma Acute Care Surg 72: 1404-1410, 2012.

36. Watters TS, Browne JA, Orlando LA, Wellman SS, Urbaniak JR and Bolognesi MP: Cost-effectiveness analysis of free vascularized fibular grafting for osteonecrosis of the femoral head. J Surg Orthop Adv 20: 158-167, 2011. 
37. Kawate K, Yajima H, Sugimoto K, et al: Indications for free vascularized fibular grafting for the treatment of osteonecrosis of the femoral head. BMC Musculoskelet Disord 8: 78, 2007.

38. Yin S, Zhang C, Jin D, Chen S, Sun Y and Sheng J: Treatment of osteonecrosis of the femoral head in lymphoma patients by free vascularised fibular grafting. Int Orthop 35: 1125-1130, 2011

39. Feng Y, Wang S, Jin D, et al: Free vascularised fibular grafting with OsteoSet ${ }^{\circledR} 2$ demineralised bone matrix versus autograft for large osteonecrotic lesions of the femoral head. Int Orthop 35: 475-481, 2011

40. Cohen R: A porous tantalum trabecular metal: basic science. Am J Orthop (Belle Mead NJ) 31: 216-217, 2002

41. Stronach BM, Duke JN, Rozensweig SD and Stewart RL: Subtrochanteric femur fracture after core decompression and placement of a tantalum strut for osteonecrosis of the femoral head. J Arthroplasty 25: 1168.e5-1168.e7, 2010.

42. Aldegheri R, Taglialavoro $G$ and Berizzi A: The tantalum screw for treating femoral head necrosis: rationale and results. Strategies Trauma Limb Reconstr 2: 63-68, 2007.

43. Varitimidis SE, Dimitroulias AP, Karachalios TS, Dailiana ZH and Malizos KN: Outcome after tantalum rod implantation for treatment of femoral head osteonecrosis: 26 hips followed for an average of 3 years. Acta Orthop 80: 20-25, 2009.

44. Schnieders MJ, Dave SB, Morrow DE, Heiner AD, Pedersen DR and Brown TD: Assessing the accuracy of a prototype drill guide for fibular graft placement in femoral head necrosis. Iowa Orthop J: 58-63, 1997.

45. Liu B, Sun W, Yue D, Li Z and Guo W: Combined tantalum implant with bone grafting for the treatment of osteonecrosis of the femoral head. J Invest Surg 26: 158-162, 2013.

46. Samara S, Dailiana Z, Chassanidis C, Koromila T, Papatheodorou L, Malizos KN and Kollia P: Expression profile of osteoprotegerin, RANK and RANKL genes in the femoral head of patients with avascular necrosis. Exp Mol Pathol 96 9-14, 2014.

47. Fan M, Jiang WX, Wang AY, Wang Y, Peng J, Zhang L, Xu WJ and Lu SB: Effect and mechanism of zoledronate on prevention of collapse in osteonecrosis of the femoral head. Zhongguo Yi Xue Ke Xue Yuan Xue Bao 34: 330-336, 2012 (In Chinese).

48. Wang C, Wang X, Xu XL, Yuan XL, Gou WL, Wang AY, Guo QY, Peng J and Lu SB: Bone microstructure and regional distribution of osteoblast and osteoclast activity in the osteonecrotic femoral head. PLoS One 9: e96361, 2014.

49. Wang CJ: Extracorporeal shockwave therapy in musculoskeletal disorders. J Orthop Surg Res 7: 11, 2012.

50. Yin TC, Wang CJ, Yang KD, Wang FS and Sun YC: Shockwaves enhance the osteogenetic gene expression in marrow stromal cells from hips with osteonecrosis. Chang Gung Med J 34: 367-374, 2011

51. Ludwig J, Lauber S, Lauber HJ, Dreisilker U, Raedel R and Hotzinger H: High-energy shock wave treatment of femoral head necrosis in adults. Clin Orthop Relat Res 387: 119-126, 2001.

52. Wang CJ, Wang FS, Huang CC, Yang KD, Weng LH and Huang HY: Treatment for osteonecrosis of the femoral head: comparison of extracorporeal shock waves with core decompression and bone-grafting. J Bone Joint Surg Am 87: 2380-2387, 2005.

53. Alves EM, Angrisani AT and Santiago MB: The use of extracorporeal shock waves in the treatment of osteonecrosis of the femoral head: a systematic review. Clin Rheumatol 28 $1247-1251,2009$

54. Turner DA, Templeton AC, Selzer PM, Rosenberg AG and Petasnick JP: Femoral capital osteonecrosis: MR finding of diffuse marrow abnormalities without focal lesions. Radiology 71: 135-140, 1989

55. Hofmann S, Schneider W, Breitenseher M, Urban M and PlenkH Jr: 'Transient osteoporosis' as a special reversible form of femur head necrosis. Orthopade 29: 411-419, 2000 (In German).

56. Yang JC, Pan ZY, Gu H, Li N, Qian XJ, Zhai RY, Wu LH and Gao CJ: Quantitative study of therapeutic efficacy on early intervention of hyperbaric oxygen to model of steroid-induced avascular osteonecrosis of femoral head by multi-slice perfusion imaging. Zhonghua Yi Xue Za Zhi 88: 3210-3216, 2008 (In Chinese).

57. Camporesi EM, Vezzani G, Bosco G, Mangar D and Bernasek TL: Hyperbaric oxygen therapy in femoral head necrosis. J Arthroplasty 25: 118-123, 2010.

58. Reis ND, Schwartz O, Militianu D, et al: Hyperbaric oxygen therapy as a treatment for stage-I avascular necrosis of the femoral head. J Bone Joint Surg Br 85: 371-375, 2003.
59. Ficat P, Arlet J, Vidal R, Ricci A and Fournial JC: Therapeutic results of drill biopsy in primary osteonecrosis of the femoral head (100 cases). Rev Rhum Mal Osteoartic 38: 269-276, 1971 (In French)

60. Lieberman JR, Conduah A and Urist MR: Treatment of osteonecrosis of the femoral head with core decompression and human bone morphogenetic protein. Clin Orthop Relat Res 429: 139-145, 2004

61. Lieberman JR: Core decompression for osteonecrosis of the hip. Clin Orthop Relat Res 418: 29-33, 2004

62. Mont MA, Ragland PS and Etienne G: Core decompression of the femoral head for osteonecrosis using percutaneous multiple small-diameter drilling. Clin Orthop Relat Res 429: 131-138, 2004

63. Steinberg ME, Brighton CT, Corces A, et al: Osteonecrosis of the femoral head. Results of core decompression and grafting with and without electrical stimulation. Clin Orthop Relat Res 249: 199-208, 1989.

64. Trancik T, Lunceford E and Strum D: The effect of electrical stimulation on osteonecrosis of the femoral head. Clin Orthop Relat Res 256: 120-124, 1990.

65. Steinberg ME, Brighton CT, Bands RE and Hartman KM: Capacitive coupling as an adjunctive treatment for avascular necrosis. Clin Orthop Relat Res 261: 11-18, 1990.

66. Steinberg ME: Core decompression of the femoral head for avascular necrosis: indications and results. Can J Surg 38 (Suppl 1): S18-S24, 1995.

67. Marker DR, Seyler TM, Ulrich SD, Srivastava S and Mont MA: Do modern techniques improve core decompression outcomes for hip osteonecrosis? Clin Orthop Relat Res 466: 1093-1103, 2008.

68. Rackwitz L, Eden L, Reppenhagen S, et al: Stem cell- and growth factor-based regenerative therapies for avascular necrosis of the femoral head. Stem Cell Res Ther 3: 7, 2012.

69. Helbig L, Simank HG, Kroeber M, Schmidmaier G, Grützner PA and Guehring T: Core decompression combined with implantation of a demineralised bone matrix for non-traumatic osteonecrosis of the femoral head. Arch Orthop Trauma Surg 132: 1095-1103, 2012.

70. Liu Y, Liu S and Su X: Core decompression and implantation of bone marrow mononuclear cells with porous hydroxylapatite composite filler for the treatment of osteonecrosis of the femoral head. Arch Orthop Trauma Surg 133: 125-133, 2013.

71. Wang W, Liu L, Dang X, Ma S, Zhang M and Wang K: The effect of core decompression on local expression of BMP-2, PPAR- $\gamma$ and bone regeneration in the steroid-induced femoral head osteonecrosis. BMC Musculoskelet Disord 13: 142, 2012.

72. Xie XH, Wang XL, He YX, et al: Promotion of bone repair by implantation of cryopreserved bone marrow-derived mononuclear cells in a rabbit model of steroid-associated osteonecrosis Arthritis Rheum 64: 1562-1571, 2012

73. Wei BF and Ge XH: Treatment of osteonecrosis of the femoral head with core decompression and bone grafting. Hip Int 21: 206-210, 2011

74. Li W, Sakai T, Nishii T, et al: Distribution of TRAP-positive cells and expression of HIF-1 $\alpha$, VEGF, and FGF-2 in the reparative reaction in patients with osteonecrosis of the femoral head. J Orthop Res 27: 694-700, 2009.

75. Zaidi M, Sun L, Robinson LJ, et al: ACTH protects against glucocorticoid-induced osteonecrosis of bone. Proc Natl Acad Sci USA 107: 8782-8787, 2010

76. Gao YS, Wang HF, Ding H and Zhang CQ: A novel rat model of osteonecrosis of the femoral head induced by periarticular injection of vascular endothelial growth factor receptor 2 antibody. J Surg Res 183: e1-e5, 2013.

77. Zhang C, Li Y, Cornelia R, Swisher S and Kim H: Regulation of VEGF expression by HIF-1 $\alpha$ in the femoral head cartilage following ischemia osteonecrosis. Sci Rep 2: 650, 2012.

78. Trueta J and Buhr AJ: The vascular contribution to osteogenesis. V. The vasculature supplying the epiphysial cartilage in rachitic rats. J Bone Joint Surg Br 45: 572-581, 1963.

79. Cao K, Huang W, An H, Jiang DM, Shu Y and Han ZM: Deproteinized bone with VEGF gene transfer to facilitate the repair of early avascular necrosis of femoral head of rabbit. Chin J Traumatol 12: 269-274, 2009.

80. Hang D, Wang Q, Guo C, Chen Z and Yan Z: Treatment of osteonecrosis of the femoral head with VEGF165 transgenic bone marrow mesenchymal stem cells in mongrel dogs. Cells Tissues Organs 195: 495-506, 2012.

81. Rizzoli R: Bisphosphonates treatment in patients with osteoporosis. Ther Umsch 69: 173-181, 2012 (In German). 
82. Gou W, Wang X, Peng J, et al: Controlled delivery of zoledronate improved bone formation locally in vivo. PLoS One 9: e91317, 2014.

83. Hayes WC, Shea M and Rodan GA: Preclinical evidence of normal bone with alendronate. Int J Clin Pract Suppl 101: 9-13, 1999.

84. Agarwala S, Sule A, Pai BU and Joshi VR: Study of alendronate in avascular necrosis of bone. J Assoc Physicians India 49: 949-950, 2001.

85. Agarwala S, Sule A, Pai BU and Joshi VR: Alendronate in the treatment of avascular necrosis of the hip. Rheumatology (Oxford) 41: 346-347, 2002.

86. Peled E, Bejar J, Barak M, Orion E and Norman D: Core decompression and alendronate treatment of the osteonecrotic rat femoral head: computer-assisted analysis. Int J Exp Pathol 94: 212-216, 2013

87. Agarwala S, Jain D, Joshi VR and Sule A: Efficacy of alendronate, a bisphosphonate, in the treatment of AVN of the hip. A prospective open-label study. Rheumatology (Oxford) 44 352-359, 2005 .

88. Wong PK, Borromeo GL and Wark JD: Bisphosphonate-related osteonecrosis of the jaw in non-malignant bone disease. Rheumatol Int 33: 2189-2198, 2013.
89. Outeiriño-Fernández A: Osteonecrosis of the jaw associated with bisphosphonate therapy in primary osteoporosis. Review of the literature. An Sist Sanit Navar 36: 87-97, 2013 (In Spanish).

90. Chiu WY, Lee JJ and Tsai KS: Atypical femoral fractures shortly after osteonecrosis of the jaw in a postmenopausal woman taking alendronate for osteoporosis. J Clin Endocrinol Metab 98: E723-E726, 2013.

91. Conte Neto N, Spolidorio LC, Andrade CR, S Bastos A, Guimarães M and Marcantonio E Jr: Experimental development of bisphosphonate-related osteonecrosis of the jaws in rodents. Int J Exp Pathol 94: 65-73, 2013.

92. Arranz Caso JA,Flores BallesterE,Ngo Pombe S,LópezPizarro V, Dominguez-Mompello JL and Restoy Lozano A: Bisphosphonate related osteonecrosis of the jaw and infection with Actinomyces. Med Clin (Barc) 139: 676-680, 2012 (In Spanish).

93. Schaudinn C, Gorur A, Webster P, et al: Quantification by energy dispersive X-ray spectroscopy of alendronate in the diseased jaw bone of patients with bisphosphonate-related jaw osteonecrosis. Oral Surg Oral Med Oral Pathol Oral Radiol 114: 480-486, 2012. 94. Bocanegra-Pérez MS, Vicente-Barrero M, Sosa-Henríquez M, et al: Bone metabolism and clinical study of 44 patients with bisphosphonate-related osteonecrosis of the jaws. Med Oral Patol Oral Cir Bucal 17: e948-e955, 2012. 